\title{
NOTE ON THE VORTEX DENSITY IN ROTATING HELIUM II
}

\author{
D. STAUFFER \\ Max-Planck-Institut für Physik und Astrophysik, Munich, Germany \\ and \\ F. POBELL, W. SCHOE PE \\ Physik Department der Technischen Hochschule. Munich, Germany
}

Received 8 March 1968

\begin{abstract}
Computer calculations show that there exist no large stable deviations from the homogeneous vortex density in an annulus or cylinder; the solutions of Masson and Tien are unstable.
\end{abstract}

Masson and Tien [1] have recently calculated in a continuum approximation the vortex density in a rotating annulus filled with $\mathrm{He} I I$. In addition to the energetically most favourable homogeneous distribution they found solutions with a spatially oscillating vortex density. For small oscillation amplitudes the distance between two maxima of vortex density is only of the order of the vortex mean spacing $s=(\kappa / 2 \Omega)^{\frac{1}{2}},(\kappa=h / m, \Omega=$ angular velocity). Therefore these "small-amplitude" solutions are not different from the homogeneous solution. The oscillations with large amplitudes and greater distances between two peaks in the vortex density remain to be investigated. We will generalize the solutions of this type given in ref. 1 and discuss their stability.

In such equilibrium configurations with widely separated narrow peaks each peak represents a ring composed of vortices with spacing $\ll s$; the distance between the different concentric rings is $\gg s$. It was shown by exact computer calculations without averiging [2] that vortices always tend to form a system of concentric rings. In the energetically mostly favoured configurations the spacing of both the different rings and the vortices within each ring is $\approx s$.

In order to find other equilibrium configurations we assume a system of rings, each with $N_{i}$ vortices and a radius $R_{i}$. We treat the different rings separately, but approximate the interaction between them by smearing out the discrete vortex structure within each ring. This means an "exact" calculation in the radial direction and an average calculation along the circumferences of the rings. Thus each ring produces a zero velocity inside, and the velocity $N_{i} \kappa / 2 \pi r$ outside. The self-in- duced velocity $\left(N_{i}-1\right) \kappa / 4 \pi R_{i}$ at its radius is nearly the average of the inner and outer velocities. At equilibrium each ring is at rest in the rotating frame; this condition yields the approximate relation between $N_{i}$ and $R_{i}$ :

$$
\Omega R_{i}=\left(\Gamma_{1}+\frac{1}{2}\left(N_{i}-1\right) \kappa\right) / 2 \pi R_{i}
$$

Here $\Gamma_{1}$ is the total circulation within the $i$-th ring, $\Gamma_{1}=\Gamma_{0}+\kappa \sum_{k=1}^{i-1} N_{k}$, where $\Gamma_{\mathrm{o}}$ is the circulation around the inner cylinder in an annulus or around the center vortex in a cylinder. For example, in a cylinder with 37 vortices two of the stable configurations are given by $N_{i}=$ $=6,12,18$ and $N_{i}=7,12,17$ [2, fig. 1]. In the most extreme case the center vortex might be surrounded by a single ring with 36 vortices: $N_{i}=0,36,0$. Similarly, for larger vortex numbers $N_{i}$ may be varied rather arbitrarily as long as all $R_{i}$ lie within the bucket. Therefore eq. (1) with nearly arbitrary changes of $R_{i}$ seems to be an appropriate generalization of the solution of ref. 1 (nearly periodic variations of the vortex density).

However, not all of these equilibrium configurations are stable against small (not necessarily axially symmetric) perturbations. A ring of more than 6 vortices without center vortex is unstable [3]; with center vortex the boundary is 9 . A system of well separated rings with closely spaced vortices is similar to a system of classical vortex sheets which are unstable $[4,5]$. If we assume that the vortex rings are well separated we can used eq. (14) of ref. 3 to show that each radius $R_{i}$ of the homogeneous solution can be enlarged in stable systems only by $\Delta R_{i} \lesssim 0.1 \mathrm{~s}$. 
This shows, that the large deviations $\left(\Delta R_{i} \gg s\right)$ suggested by ref. 1 are unstable. Perhaps this limit for $\Delta R_{i}$ is quantitatively incorrect because now the rings are nog well separated. Hence, we performed exact computer calculations using the iteration program of ref. 2. Neglecting image forces the following cases were solved: the annulus ( 2 rings) with 30,42 and 54 vortices $\left(\Gamma_{\mathrm{o}} / \kappa=7,19\right.$ and 37 , respectively) and the cylinder with 19,37 and 61 vortices $(2,3$ and 4 rings, respectively). The calculations show that the $R_{i}$ from eq. (1) are accurate within $0.02 s$ if $N_{i} \geqslant 5$. Shifting some vortices among the different rings we found the configurations to become unstable if the greatest deviation $\left|\Delta R_{i}\right| / s$ exceeds a value between 0.07 and 0.08 . This number agrees with the above estimate and shows no tendency to increase with increasing vortex number. Therefore the radii of all vortex rings can be changed at most by a small fraction of the vortex mean spacing from its energetically most favourable value; this means that the vortex density is always homogenous.

We thank Dr. Masson and Prof. Tien for correspondence. One of us (D.S.) is indebted to the Max-Planck-Institut for a grant.

\section{NEW FORM FOR THE THERMODYNAMIC POTENTIAL OF He II NEAR T D. J. AMIT \\ Department of Theoretical Physics, Hebrew University, Jerusalem, Israel}

1. B. Masson and C. L. Tien, Phys. Rev. 165 (1968) 300.

2. D. Stauffer and A. L. Fetter, Phys. Rev., to be published.

3. T. H. Havelock, Phil. Mag. (VII) 11 (1931) 617.

4. A. Michalke and A. Timme, J. Fluid Mech. 29 (1967) 647.

5. J.R. Weske and T. M. Rankin, Phys. Fluids 6 (1963) 1397 , fig. 5 .

Received 4 March 1968

A new thermodynamic pctential function is presented for temperatures just below $T_{\lambda}$ which reproduces the $\left(T_{\lambda}-T\right) \frac{2}{3}$ behavior of $\rho_{\mathrm{S}}$ and the critical velocity $v_{\mathrm{C}}$.

Accurate measurements of the superfluid density $\rho_{\mathrm{S}}[1]$ as one approaches $T_{\lambda}$ from below re veal a behavior of the form $\rho_{\mathrm{S}}=1.44 \rho(T \lambda-T)^{\frac{2}{3}}$. This implies, immediately, that the theory of Ginzburg and Pitaevskii [2] which predicts a linear behavior of $\rho_{\mathrm{S}}$ as a function of $T \lambda-T$ has to be amended. This has been recently taken up by Mamaladze [3] who keeps the Landau [4,5] expansion of the thermodynamic potential but changes the temperature dependence of the coefficients in the expansion. The expansion is carried out in powers of the order parameter $\psi$ which is connected to the superfluid number density $\rho_{\mathbf{S}}$ via $\rho_{\mathbf{S}}=|\psi| 2 *$. If the system is at rest we

$* \psi$ is connected to Josephson's $\widetilde{\psi}$ via $\psi=m^{\frac{1}{2}} \widetilde{\psi}$. It would be proportional to Josephson's $\psi$ if $\eta$ turned out to be zero. can take $\psi$ to be real and positive. We then write Mamladze's [3] result in the following form:

$$
\Phi(P, T)=\Phi_{\mathrm{I}}(P, T)+A \psi^{2}+B \psi^{4},
$$

with $A=-\alpha\left(T_{\lambda}-T\right) \frac{4}{3}, B=\beta\left(T_{\lambda}-T\right) \frac{2}{3}, \alpha, \beta>0$, $\Phi_{\mathrm{I}}$ is the thermodynamic potential of HeI. The equilibrium value of $\psi$ or $\rho_{\mathbf{S}}$ is determined by minimizing $\Phi$ with respect to $\psi, \alpha>0$ follows from the requirement that $\psi=0$ will not be minimum at $T<T_{\lambda}$ even though it solves $\partial \Phi / \partial \psi=0$. In the old theory $[2,4,5] A$ was linear in $T_{\lambda}-T$ and $B$ a constant. The sign of $B$ was dictated by the requirement that $\psi=0$ will be a stable minimum at $T=T \lambda$. This last requirement cannot be satisfied with eq. (1) [3] since both coefficients vanish at $T=T \lambda$. In this case $\rho_{\mathrm{S}}$ at equilibrium is $(\alpha / 2 \beta)\left(T_{\lambda}-T\right)^{\frac{2}{3}}$ and to make $\rho_{\mathrm{S}}>0$ we must 